\title{
Comparing Pioglitazone to Insulin with Respect to Cancer, Cardiovascular and Bone Fracture Endpoints, Using Propensity Score Weights
}

\author{
Carlos Vallarino • Alfonso Perez • Gregory Fusco • \\ Huifang Liang • Morgan Bron · Sudhakar Manne • \\ Guiandre Joseph $\cdot$ Shawn Yu
}

Published online: 24 July 2013

(c) The Author(s) 2013. This article is published with open access at Springerlink.com

\begin{abstract}
Background Diabetes is an important global disease, associated with significant morbidity and an increased risk of death due to chronic end-organ complications. The thiazolidinediones, used mainly as third-line agents in type 2 diabetes mellitus (T2DM), have been associated with some safety concerns, such as an increased risk of bladder cancer, an increased risk of bone fracture and heterogeneous effects on cardiovascular events.

Objective This study aimed to evaluate safety data on pioglitazone for several outcomes and examine them in context with each other as well as with insulin, another third-line treatment for T2DM.

Methods This retrospective cohort study extracted data from May 1, 2000 until June 30, 2010, from the i3 InVision Data Mart ${ }^{\mathrm{TM}}$ database. To adjust for the testing of multiple hypotheses, the Holm method was applied to endpoints representing potential harm from pioglitazone treatment, separately from those representing potential benefit from pioglitazone. The study population included patients with $\mathrm{T} 2 \mathrm{DM} \geq 45$ years old who were new users of either pioglitazone or insulin. Key outcomes were incident cases of a composite of myocardial infarction (MI) or stroke requiring hospitalization; bone fracture requiring hospitalization; bladder cancer; and a composite of nine other selected cancers. Kaplan-Meier curves were generated and hazard ratios
\end{abstract}

C. Vallarino $(\bowtie) \cdot$ A. Perez $\cdot$ G. Fusco $\cdot$ H. Liang $\cdot$ S. Manne

G. Joseph

Takeda Global Research and Development Center, Inc.,

One Takeda Parkway, Deerfield, IL 60015, USA

e-mail: carlos.vallarino@takeda.com

M. Bron · S. Yu

Takeda Pharmaceuticals International, One Takeda Parkway,

Deerfield, IL 60015, USA
(HRs) for pioglitazone versus insulin were estimated from Cox proportional hazards models adjusted with inverse probability of treatment weights derived from propensity scores.

Results A total of 56,536 patients (pioglitazone group 38,588 ; insulin group 17,948) qualified for the study. The mean follow-up was 2.2 years for pioglitazone and 1.9 years for insulin patients. Weighted survival analysis of the composite of MI and stroke, as well as the composite of nine cancers, yielded significant differences in favour of pioglitazone. For the composite of MI and stroke, the HR for pioglitazone versus insulin was 0.44 (95\% confidence interval $[\mathrm{CI}] 0.39-0.50, p<0.0001)$. Modelling of the composite of nine selected cancers produced an HR of 0.78 (95\% CI $0.71-0.85, p<0.0001)$. A non-statistically significant difference in favour of pioglitazone was observed in the incidence rate of bone fracture requiring hospitalization (HR 0.86, $95 \%$ CI $0.74-1.01, p=0.058$ ). For bladder cancer, the overall incidence rates were relatively low and showed no significant difference between the two groups; the HR for pioglitazone versus insulin was 0.92 (95\% CI 0.63-1.33, $p=0.64$ ).

Conclusion Compared with insulin, pioglitazone was associated with a significant reduction in the risk of MI and stroke requiring hospitalization, and a significant reduction in the risk of other selected cancers. While pioglitazone treatment may be linked with a lower risk of bladder cancer and bone fracture relative to insulin, these differences were not statistically significant.

\section{Introduction}

The seventh leading cause of death in the US, type 2 diabetes mellitus (T2DM) increases the risk of mortality from causes such as vascular disease-in particular, coronary 
heart disease [1]. A recent publication showed that after adjustment for age, sex, smoking status and body mass index, diabetics, compared with non-diabetics, have hazard ratios (HRs) of 1.25 for death from cancer, 2.32 for death from vascular causes and 1.73 for death from other causes [2]. This same study also found that diabetes was associated with substantial premature mortality from infectious diseases and degenerative conditions.

The thiazolidinediones (TZDs), rosiglitazone and pioglitazone, are synthetic ligands for peroxisome proliferatoractivated receptors (PPARs) that alter the transcription of genes influencing carbohydrate and lipid metabolism [3]. Both TZDs improve insulin sensitivity through their action at PPAR gamma receptors-with similar effects on glucose levels - but pioglitazone demonstrates a different effect on lipid metabolism [4, 5] and further has been associated with a reduction in the risk of hospitalization for acute myocardial infarction (AMI) compared with rosiglitazone [6]. Results from the PROactive (PROspective pioglitAzone Clinical Trial In macroVascular Events) study have shown that pioglitazone, in conjunction with standard treatments for diabetes and cardiovascular (CV) conditions, can reduce the risk of the composite endpoint of all-cause mortality, non-fatal myocardial infarction (MI) and stroke [7]. Regarding insulin, attention has been drawn to the potential link between hyperinsulinization and CV events [8].

Animal toxicity studies have both suggested a possible increased cancer risk in multiple organs in association with a wide variety of PPAR modulators such as pioglitazone [9] and, in contrast, attributed PPAR gamma activators with inhibitory effects on tumour development [10]. Few clinical trials or epidemiological data have provided information on PPAR modulators and the risk of cancer in association with their use [11]; conversely, insulin has been studied for decades and is posited to exhibit mitogenic effects on cancer tumours [12]. In addition, pioglitazone has been associated with bone fracture in postmenopausal women [13] but, again, clinical studies to date on PPAR modulators and bone fracture have been rare.

Requests from regulatory bodies have led to research, initiated in 2003, to corroborate a possible increased risk of cancer with pioglitazone, addressing cancer in general and bladder cancer in particular. Interim results have shown no evidence of an association between the use of pioglitazone and the risk of cancer at the ten most common cancer sites, compared with use of other oral anti-diabetic agents [11]. Moreover, any use of pioglitazone was not associated with an increased incidence of bladder cancer, although use for more than 2 years was weakly associated with an increased risk [14, 15]. Recently published studies have offered an array of results and inferences regarding this topic [16-18]. A public-private enterprise assembled to help improve the monitoring of drugs for safety, the Observational Medical Outcomes Partnership (OMOP), has found that the choices made during an observational study, regarding the design, database, comparator and covariates, can drastically alter results of research focusing on a specific drug/outcome pair [19].

Pioglitazone and insulin have never been directly compared in any published literature, even though it is a natural comparison, given that both treatments occur at roughly the same stage of the progression of diabetes as a disease-as shown, for example, in the Comprehensive Diabetes Management Algorithm under dual therapy and triple therapy [20]. Pioglitazone is typically used as a third-line therapy following failure of metformin and/or sulfonylurea, while insulin is commonly used after failure of mono- and dual-diabetes therapy. This observational study aims to fill that gap, by investigating and putting into context event rates for a range of outcomes, from cancer to bone fracture to cardiovascular events, for both treatments. These endpoints and their relationship to pioglitazone have been analyzed separately in the past few years; bringing them together in one study can sharpen the focus on the relative benefits and risks associated with pioglitazone.

\section{Methods}

\subsection{Study Design}

This retrospective cohort study, abiding by a documented pre-specified protocol (available from the authors upon request), extracted data from May 1, 2000 until June 30, 2010, from the $i 3$ InVision Data Mart ${ }^{\mathrm{TM}}$. The i3 database contains longitudinal health claims from approximately 47 million participants with both medical and prescription drug coverage under the United Healthcare insurance plans in the US. Because of the non-interventional nature of the study, no ethical approval or informed patient consent were sought.

\subsection{Patient Selection}

The index date was defined as the first claim date of the index medication (pioglitazone or insulin) between January 1, 2003 and December 31, 2008, and was considered the first day of follow-up. The period of at least 180 days before the index medication was defined as the baseline period. A minimum of 28 days from the index date to the end of insurance eligibility or the end of the study period (June 30, 2010)—whichever came first-comprised the follow-up period. Although the database does not specifically record death, the decease of a patient during followup would have resulted in censoring due to the patient's loss of insurance eligibility. 
The study population consisted of patients with T2DM, identified using International Classification of Diseases (ICD)-9 diagnosis codes $250 . x 0$ or $250 . x 2$, and divided into two groups: new users of any pioglitazone-containing products and new users of any insulin-containing products ( $n=716,831$ included $)$. All patients were $\geq 45$ years of age $(n=119,423$ excluded $)$, had relevant insurance enrolment periods and claims ( $n=299,736$ excluded) and met minimum requirements for the baseline and follow-up periods ( $n=133,334$ excluded). Other exclusion criteria included type 1 diabetes mellitus, type 2 gestational diabetes, diabetes insipidus or renal glycosuria $(n=23,704)$, and use of rosiglitazone-containing products at any time $(n=63,388)$. Patients who switched from one index medication to the other, or who took both drugs simultaneously, were excluded $(n=8,549)$, as were patients with only one claim for either pioglitazone or insulin within 6 months of the index date $(n=12,161)$, leaving the study with 38,588 pioglitazone patients and 17,948 insulin patients.

Also excluded from specific endpoint analyses were patients who experienced any relevant incident outcomes during the baseline period: for the cardiovascular (CV) endpoint analysis, patients with a corresponding $\mathrm{CV}$ event within 28 days prior to the index date $(n=1,200)$ were excluded; for the bone fracture endpoint analysis, patients with a bone fracture within 360 days prior to the index date $(n=1,818)$ were excluded; for any cancer endpoint analysis, patients with a diagnosis of any cancer prior to the index date $(n=6,961)$ were excluded. Allowing for a 28-day baseline exclusionary period for a $\mathrm{CV}$ event, or even a 1-year period for bone fracture, does not fit the traditional definition of incidence. However, use of such conservative exclusion criteria maximizes the number of patients in the study and the total count of events-two prime considerations.

\subsection{Statistical Analysis}

Propensity scores were used to alleviate concerns about the introduction of bias due to differences in baseline covariates between the two treatment cohorts [21, 22]. Although matching was an option, weights based on propensity scores additionally serve to allay some researchers' apprehension that matching might exclude a substantial sample size of patients whose disease experience could make a valid contribution. Considering weights based on both the standardized morbidity ratio [23] and the inverse probability of treatment (IPT) [24, 25], we opted for the latter. These weights yield cohorts that are effectively from a common population, except for the difference in the propensity score response variable-in our case, the index medications.
A wealth of baseline data included demographics (age, sex, tobacco use), use of medications (defined as any prescription claim within 180 days prior to the index date) and medical history (defined as any diagnosis claim prior to the index date). These variables were chosen based on a priori considerations of clinical significance in relation to the risks of cardiovascular events, bone fracture and bladder cancer. The final list of covariates used to estimate the propensity scores was based on a stepwise logistic regression model, with $p<0.2$ to enter the model and $p<0.05$ to remain (age and sex remained, regardless of the $p$ value). Roughly 30 of the more than 40 independent variables fitted were retained by each model.

\subsubsection{Outcome Measures}

The study focused on incident cases of four major endpoints: a composite of MI or stroke requiring hospitalization; bone fracture requiring hospitalization; bladder cancer; and a composite of the nine most common cancers, excluding bladder cancer (prostate, female breast, lung/ bronchus, pancreatic, endometrial, non-Hodgkin's lymphoma, colorectal, kidney and malignant melanoma). All ICD-9 diagnosis codes listed in Table 1 were carefully chosen in the study design stage. Validated coding algorithms for the outcomes were selected when available in the literature. For example, ICD-9 codes for incident MI and stroke have been validated against medical charts with high positive predictive values $[26,27]$. To verify the findings based on incident cases of bladder cancer, a secondary analysis was performed using additional therapy codes and procedures to confirm the presence of cancer, such as radiation treatment, chemotherapy, cystoscopy or cystectomy.

Each outcome used a unique, conservative drug residual effect period based on the clinical course of the specific disease, which was equivalent to the baseline exclusionary period: 28 days for $\mathrm{CV}$ events and 360 days for bone fracture events; there was no time limit on the occurrence of any cancer events. Hence CV and bone fracture outcomes recorded after this pre-specified period following the last day of the last prescription were deemed unrelated to treatment.

\subsubsection{Modelling and Testing}

Analyses were performed separately on the first occurrence of each major endpoint. The time to event spanned from the index date until the earliest of the following: the first event date; the last day of the drug residual effect period; or the patient's last day of insurance eligibility.

The hazard ratios of pioglitazone over insulin and $95 \%$ confidence intervals (CIs) were estimated from Cox 
Table 1 International Classification of Diseases (ICD)-9 codes for major endpoints and their components

\begin{tabular}{ll}
\hline Endpoint & ICD-9 code \\
\hline MI & $410 . x x$ \\
Stroke & $430,431,433 . x 1$ \\
& $434 . x x$ (excluding 434.x0), 436 \\
Bone fracture & $800 . x x-829 . x x$ \\
Bladder cancer & $188 . x x, 233.7$ \\
Prostate cancer & $185 . x x, 233.4$ \\
Female breast cancer & $174 . x x, 233.0$ \\
Lung cancer & $162 . x, 231.1,231.2$ \\
Pancreatic cancer & $157 . x$ (excluding 157.4) \\
Endometrial cancer & $179,182 . x$ \\
Non-Hodgkin's lymphoma & $200 . x x, 202 . x x$ \\
Colorectal cancer & $153 . x, 154.0,154.1,230.3,230.4$ \\
Kidney cancer & $189 . x$ (excluding 189.3 and 189.4) \\
Malignant melanoma & $172 . x, 232 . x$ \\
\hline
\end{tabular}

MI myocardial infarction

proportional hazards models using IPT weights. Crude event rates across time were also calculated. Unweighted Cox regression models using the propensity score as a covariate, as well as the full set of covariates, were fitted as sensitivity checks on the results from modelling with inverse probability weights, yielding very similar HRs. The proportional hazards assumption was verified through the graphical and re-sampling techniques of Lin et al. [28] and by plotting the log of cumulative hazard vs. $\log$ of time. Analyses were conducted using SAS version 9.1 software (SAS Institute, Cary, NC, USA).

To adjust for the multiplicity of hypothesis tests, a twofold approach to control the overall probability of a type I error was decided upon during the design phase of the study. The CV endpoint, representing potential benefit from pioglitazone in comparison with insulin, would be treated separately and tested at the 0.05 significance level (two-sided). The three other major endpoints, representing potential risks from pioglitazone treatment, would be adjusted for multiplicity using the Holm method [29], also at the 0.05 level.

\section{Results}

\subsection{Baseline}

Table 2 presents a summary of demographics for the 38,588 pioglitazone and 17,948 insulin users who fulfilled the inclusion and exclusion criteria. First prescriptions of pioglitazone were evenly dispensed throughout the study period, with a slight increase in later years, while one third of first prescriptions for insulin were dispensed in the last year. Differences in the medical conditions that were prevalent in the pioglitazone and insulin groups were observed. With the exceptions of hyperlipidaemia and hypertension, which were recorded in greater proportions of patients in the pioglitazone group, all other medical conditions were observed in larger percentages of patients receiving insulin.

Similarly, the majority of dispensed drugs were used by greater proportions of patients in the insulin group. The effect of the IPT weights used to balance the cohorts with respect to these differences can be seen in Table 2. Compared with the unweighted $p$ values, all of the covariatessave two at the 0.05 level, or four at the 0.10 level-were successfully balanced by the weighting technique. Given a list of more than 40 covariates, and the significant multiplicity that such a list entails, it is to be expected that some would remain unbalanced even after weighting. As a sensitivity check, these covariates were added to the Cox regressions, with no change in the treatment effect.

\subsection{Raw Incidence}

The mean follow-up time was 2.2 years in the pioglitazone group and 1.9 years in the insulin group, with some patients being followed up for as long as 7 years. The average raw incidence rates for pioglitazone and insulin for each major endpoint are shown side by side in Fig. 1.

Relative to the other endpoints, bladder cancer had substantially lower incidence rates: 113 and 152 per 100,000 person-years for pioglitazone and insulin, respectively. The cancer composite had the highest incidence rates: 1,798 for pioglitazone, 2,456 for insulin. Pioglitazone also had lower incidence rates than insulin for the composite of MI and stroke (717-2,067) and bone fracture (581-959 per 100,000 person-years).

Note that the average raw incidence rates of the composite of nine other selected cancers for both pioglitazone and insulin were 16 times higher than the respective rates of bladder cancer alone. Bone fracture and the CV composite rates ranged from 5 to 13 times the rate of bladder cancer.

\subsection{Modelling}

\subsubsection{Composite of MI and Stroke}

For the composite of MI and stroke, the HR for pioglitazone versus insulin from a Cox regression using IPT weights was 0.44 (95\% CI $0.39-0.50, p<0.0001)$ [Fig. 2a shows the Kaplan-Meier event-free probability curves]. MI and stroke endpoints were then tested individually: the HRs of pioglitazone versus insulin were 0.49 
Table 2 Patient demographics and baseline covariates

\begin{tabular}{|c|c|c|c|c|}
\hline Patient characteristic & Pioglitazone, $n=38,588$ & Insulin, $n=17,948$ & Unweighted $p$ value $^{\mathrm{a}}$ & Weighted $p$ value ${ }^{\mathrm{b}}$ \\
\hline \multicolumn{5}{|l|}{ Age } \\
\hline Mean [years (SD)] & $58.1(8.7)$ & $59.7(10.3)$ & $<0.0001$ & 0.8064 \\
\hline Median [years] & 57 & 58 & & \\
\hline $45-54$ years $[n(\%)]$ & $14,490(37.6)$ & $6,432(35.8)$ & & \\
\hline $55-64$ years $[n(\%)]$ & $16,618(43.1)$ & $6,929(38.6)$ & & \\
\hline $65-74$ years $[n(\%)]$ & $5,371(13.9)$ & $2,685(15.0)$ & & \\
\hline $75-80$ years $[n(\%)]$ & $1,325(3.4)$ & $976(5.4)$ & & \\
\hline $80+$ years $[n(\%)]$ & $784(2.0)$ & $926(5.2)$ & & \\
\hline Male sex $[n(\%)]$ & $22,981(59.6)$ & $9,504(53.0)$ & $<0.0001$ & 0.1828 \\
\hline \multicolumn{5}{|l|}{ Year of drug initiation $[n(\%)]$} \\
\hline 2003 & $4,734(12.3)$ & $1,989(11.1)$ & $<0.0001$ & 0.8908 \\
\hline 2004 & $5,292(13.7)$ & 2,137 (11.9) & & \\
\hline 2005 & $6,916(17.9)$ & $2,581(14.4)$ & & \\
\hline 2006 & $7,915(20.5)$ & 2,321 (12.9) & & \\
\hline 2007 & $5,591(14.5)$ & $2,923(16.3)$ & & \\
\hline 2008 & $8,140(21.1)$ & $5,997(33.4)$ & & \\
\hline \multicolumn{5}{|l|}{ Medical conditions $[n(\%)]$} \\
\hline Obesity & $5,798(15.0)$ & $2,831(15.8)$ & 0.0213 & 0.1356 \\
\hline Hyperlipidaemia & $30,410(78.8)$ & $11,608(64.7)$ & $<0.0001$ & 0.1308 \\
\hline MI & $1,677(4.3)$ & $1,615(9.0)$ & $<0.0001$ & 0.6195 \\
\hline Coronary heart disease & 7,576 (19.6) & 4,998 (27.8) & $<0.0001$ & 0.8043 \\
\hline Coronary revascularization & $1,482(3.8)$ & $1,201(6.7)$ & $<0.0001$ & 0.7295 \\
\hline Stroke & $3,041(7.9)$ & $2,573(14.3)$ & $<0.0001$ & 0.9425 \\
\hline Congestive heart failure & $1,846(4.8)$ & $3,029(16.9)$ & $<0.0001$ & 0.7792 \\
\hline Hypertension & $29,641(76.8)$ & $13,498(75.2)$ & $<0.0001$ & 0.8608 \\
\hline Arrhythmias & $3,737(9.7)$ & $3,378(18.8)$ & $<0.0001$ & 0.6126 \\
\hline Smoking & $3,289(8.5)$ & $2,148(12.0)$ & $<0.0001$ & 0.8784 \\
\hline Cancer & $4,171(10.8)$ & $2,790(15.5)$ & $<0.0001$ & 0.9210 \\
\hline Bone fracture & $2,231(5.8)$ & $1,423(7.9)$ & $<0.0001$ & 0.8626 \\
\hline Renal impairment & $2,503(6.5)$ & $3,112(17.3)$ & $<0.0001$ & 0.7158 \\
\hline Gout & $1,737(4.5)$ & $861(4.8)$ & 0.1179 & 0.6195 \\
\hline \multicolumn{5}{|c|}{ Dispensed anti-diabetic drugs $[n(\%)]$} \\
\hline Metformin & $15,153(39.3)$ & $5,991(33.4)$ & $<0.0001$ & 0.0530 \\
\hline Sulfonylureas & $12,670(32.8)$ & $6,972(38.8)$ & $<0.0001$ & $<0.0001$ \\
\hline Meglitinides & $576(1.5)$ & $441(2.5)$ & $<0.0001$ & 0.6847 \\
\hline DPP-4 & $652(1.7)$ & $614(3.4)$ & $<0.0001$ & 0.4871 \\
\hline GLP & $356(0.9)$ & $629(3.5)$ & $<0.0001$ & 0.3002 \\
\hline Other anti-diabetic drugs & $95(0.2)$ & $135(0.8)$ & $<0.0001$ & 0.4840 \\
\hline \multicolumn{5}{|l|}{ Other dispensed drugs $[n(\%)]$} \\
\hline Nitrates & $1,206(3.1)$ & $1,067(5.9)$ & $<0.0001$ & 0.8258 \\
\hline$\beta$-blockers & $8,901(23.1)$ & $4,860(27.1)$ & $<0.0001$ & 0.7236 \\
\hline Calcium channel blockers & $6,341(16.4)$ & $3,243(18.1)$ & $<0.0001$ & 0.4789 \\
\hline Diuretics & $6,828(17.7)$ & $4,688(26.1)$ & $<0.0001$ & 0.8243 \\
\hline ACE inhibitors/ARBs & $19,289(50.0)$ & $7,840(43.7)$ & $<0.0001$ & 0.6690 \\
\hline Antilipaemic agents & $18,922(49.0)$ & $6,652(37.1)$ & $<0.0001$ & 0.1827 \\
\hline Aspirin/NSAIDs & $5,840(15.1)$ & $2,195(12.2)$ & $<0.0001$ & 0.1639 \\
\hline Anticoagulants & $977(2.5)$ & $1,070(6.0)$ & $<0.0001$ & 0.5117 \\
\hline Antiplatelets & $1,186(3.1)$ & $851(4.7)$ & $<0.0001$ & 0.5619 \\
\hline
\end{tabular}


Table 2 continued

\begin{tabular}{lcccc}
\hline Patient characteristic & Pioglitazone, $n=38,588$ & Insulin, $n=17,948$ & ${\text { Unweighted } p \text { value }^{\mathrm{a}}}^{\text {Weighted } \text { value }^{\mathrm{b}}}$ \\
\hline Anti-epileptic agents & $2,029(5.3)$ & $1,664(9.3)$ & $<0.0001$ & 0.9523 \\
SSRIs & $3,832(9.9)$ & $2,323(12.9)$ & $<0.0001$ & 0.8897 \\
Lithium & $73(0.2)$ & $33(0.2)$ & 0.8919 & 0.2322 \\
Proton pump inhibitors & $4,872(12.6)$ & $2,831(15.8)$ & $<0.0001$ & 0.3612 \\
Bisphosphonates & $686(1.8)$ & $355(2.0)$ & 0.0993 & 0.0480 \\
Fluoride & $11(0.0)$ & $7(0.0)$ & 0.5150 & 0.9052 \\
Aromatase inhibitors & $122(0.3)$ & $76(0.4)$ & 0.0444 & 0.7692 \\
SERMs & $258(0.7)$ & $65(0.4)$ & $<0.0001$ & 0.8935 \\
Parathyroid hormone analogues & $54(0.1)$ & $54(0.3)$ & $<0.0001$ & 0.0662 \\
Immunosuppressors & $4,823(12.5)$ & $3,502(19.5)$ & $<0.0001$ & 0.9309 \\
Estrogens (systemic) & $1,644(4.3)$ & $589(3.3)$ & $<0.0001$ & 0.7314 \\
\hline ACE
\end{tabular}

$A C E$ angiotensin-converting enzyme, $A R B$ angiotensin receptor blocker, $D P P-4$ dipeptidyl peptidase-4, GLP glucagon-like peptide, $M I$ myocardial infarction, NSAID non-steroidal anti-inflammatory drug, SD standard deviation, SERM selective estrogen receptor modulator, SSRI selective serotonin reuptake inhibitor

${ }^{\text {a }}$ Cohort comparison based on a $\chi^{2}$ test for categorical variables and analysis of variance for continuous variables

${ }^{b}$ Cohort comparison weighted by inverse probability of treatment. Weights were derived from propensity scores adjusted for all baseline variables (age was a continuous covariate)

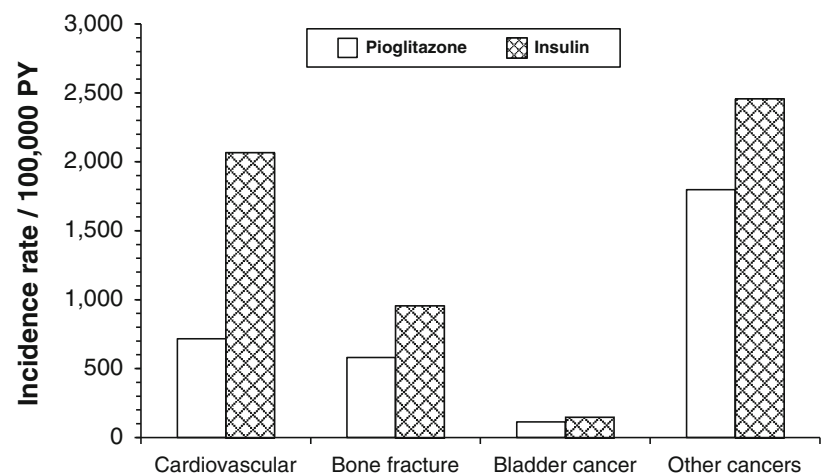

Fig. 1 Raw incidence rates for the four major study endpoints, illustrating the relatively low incidence of bladder cancer and the differences in incidence between pioglitazone and insulin. $P Y$ personyears

(95\% CI 0.41-0.57, $p<0.0001)$ for MI and 0.37 (95\% CI $0.31-0.45, p<0.0001)$ for stroke. The incidence rates, HRs and $p$ values by endpoint are reported in Table 3 .

A plot of the raw incidence rates of the composite of MI and stroke by year is shown in Fig. $2 b$. Over the first 6 years, incidence rates were greater in the insulin group; in year 7, the incidence of the CV composite was greater in the pioglitazone group, although the sample size for that year was very small (pioglitazone $n=115$, insulin $n=50$ ). The vertical bars at each year represent pointwise $95 \%$ CIs-derived from the Poisson distribution-to illustrate the inherent variability in the data, which was especially high in later years when the sample sizes were drastically reduced. To preserve a uniform, reasonable rate scale for all endpoints, the graphs cut off the upper bounds of some confidence intervals at 3,000.

\subsubsection{Multiple Hypothesis Tests for Cancers and Bone Fracture}

The three $p$ values corresponding to the primary endpoints representing potential risks from pioglitazone treatment were tested in order according to the Holm method. The smallest belonged to the cancer composite and was tested first. This HR was 0.78 (95\% CI 0.71-0.85), with a $p$ value below 0.0001 - highly statistically significant and well below $0.05 / 3$ or 0.0167 (the allotted significance level). This enabled hypothesis testing to continue. The second smallest $p$ value belonged to bone fracture with hospitalization, with an HR of 0.86 (95\% CI 0.74-1.01) and a $p$ value of 0.058 . The $p$ value was larger than 0.025 (the allowed significance level) and therefore not significant. Thus, the testing procedure was stopped, and the $p$ value for bladder cancer was automatically declared not significant.

\subsubsection{Cancer Composite}

The Kaplan-Meier curves for the composite of nine selected cancers appear in Fig. 3a. The raw incidence rate of the composite cancer endpoint was greater in the insulin group in each of the first 5 years of follow-up (Fig. 3b). As reported under 'Composite of MI and Stroke', the later years of observation were prone to progressively higher variability due to reduced sample sizes. 
Fig. 2 a Kaplan-Meier eventfree probability curves for time to myocardial infarction (MI) or stroke; $\mathbf{b}$ raw incidence rates of the composite of MI and stroke. The vertical bars at each year represent pointwise $95 \%$ confidence intervals (CIs) derived from the Poisson distribution, cut off at 3,000 in order to preserve a uniform, reasonable rate scale for all endpoints. $P Y$ person-years
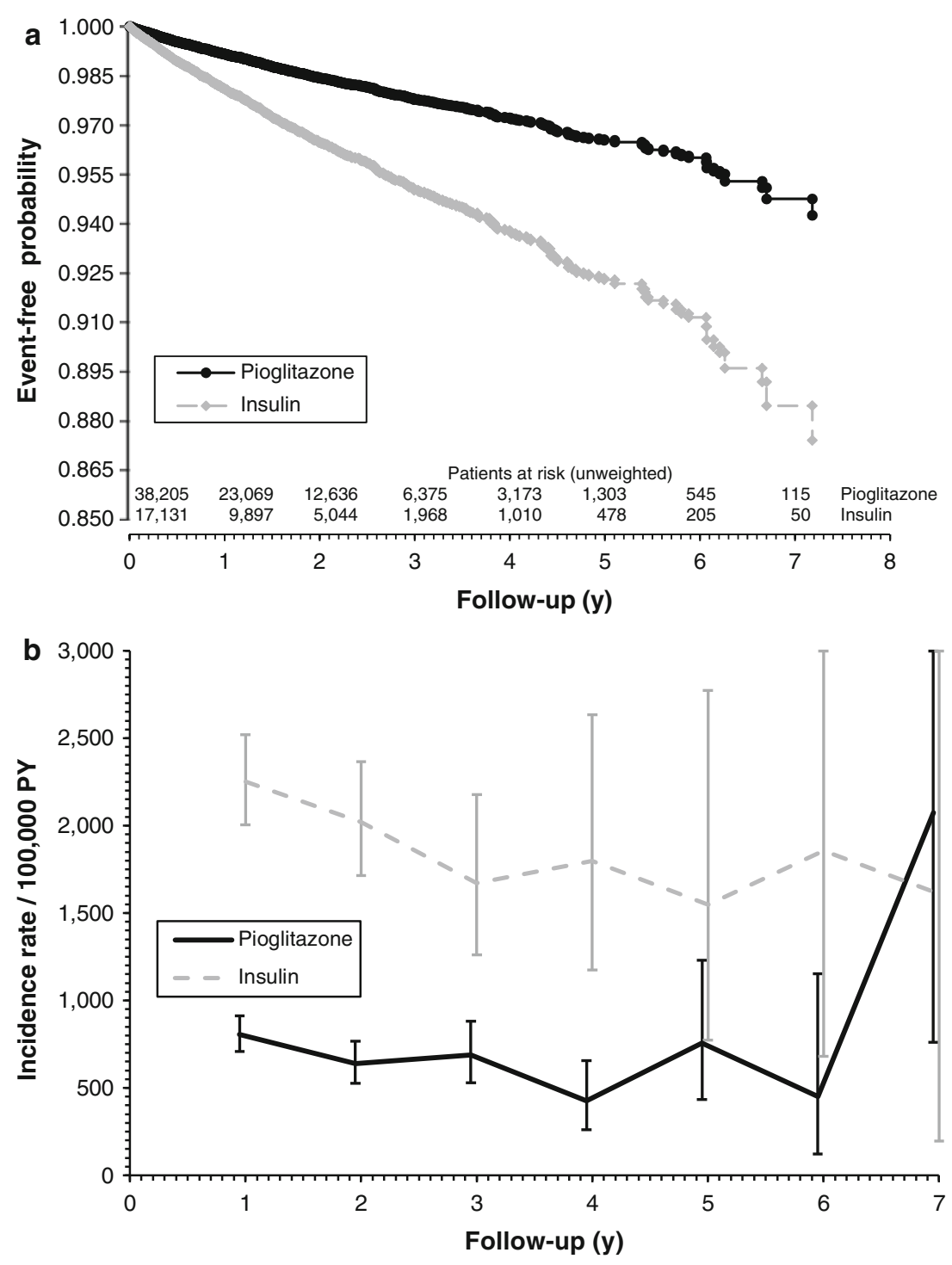

\subsubsection{Bone Fracture}

The incidence rates of bone fracture requiring hospitalization were largely similar between the two groups throughout the follow-up period.

\subsubsection{Bladder Cancer}

Based on a scant 84 events from the pioglitazone arm and 44 from the insulin arm, the event-free probability of bladder cancer was similar in the two groups and remained above 0.99 at 5.8 years, the time of the last event (not shown). The HR for pioglitazone versus insulin was 0.92 (95\% CI $0.63-1.33, p=0.64$ ). The incidence rates of bladder cancer were low and similar in the pioglitazone and insulin groups (Fig. 4). Furthermore, there was no evidence of an increasing risk of bladder cancer over time due to the use of pioglitazone. A secondary analysis, designed to further confirm the presence of bladder cancer through additional therapy codes and procedures, produced similar results (Table 3 ).

\subsubsection{Individual Cancers and Other Secondary Endpoints}

The HRs for secondary endpoints, such as individual cancers and bone fracture not requiring hospitalization, are also shown in Table 3. Patients in the pioglitazone group showed markedly lesser risks of prostate cancer, lung cancer, pancreatic cancer and colorectal cancer, compared with those in the insulin group. Female breast cancer, endometrial cancer and kidney cancer, as well as malignant melanoma, showed similar incidence rates in both groups, while the HR for non-Hodgkin's lymphoma favoured the insulin group. However small these $p$ values were, none 
Table 3 Raw average incidence rate and adjusted hazard ratios (HRs) by endpoint

\begin{tabular}{|c|c|c|c|c|}
\hline \multirow[t]{2}{*}{ Outcome } & \multicolumn{2}{|c|}{$\begin{array}{l}\text { Incidence rate per } 100,000 \text { person-years } \\
\text { (number of events) }\end{array}$} & \multirow[t]{2}{*}{$\begin{array}{l}\text { Adjusted HR } \\
(95 \% \mathrm{CI})^{\mathrm{a}}\end{array}$} & \multirow[t]{2}{*}{$p$ value } \\
\hline & Pioglitazone, $n=38,588$ & Insulin, $n=17,948$ & & \\
\hline \multicolumn{5}{|l|}{ Primary endpoints } \\
\hline Composite of MI and stroke with hospitalization & $717(472)$ & $2,067(554)$ & $0.44(0.39-0.50)$ & $<0.0001$ \\
\hline Composite of nine other selected cancers & $1,798(1,305)$ & $2,456(689)$ & $0.78(0.71-0.85)$ & $<0.0001$ \\
\hline Bone fracture with hospitalization & $581(424)$ & $959(287)$ & $0.86(0.74-1.01)$ & 0.0577 \\
\hline Bladder cancer & $113(84)$ & $152(44)$ & $0.92(0.63-1.33)$ & 0.6405 \\
\hline \multicolumn{5}{|l|}{ Secondary endpoints } \\
\hline MI with hospitalization & $454(301)$ & $1,155(318)$ & $0.49(0.41-0.57)$ & $<0.0001$ \\
\hline Stroke with hospitalization & $271(180)$ & $959(265)$ & $0.37(0.31-0.45)$ & $<0.0001$ \\
\hline Bone fracture not requiring hospitalization & $2,926(2,066)$ & $4,033(1,162)$ & $0.91(0.84-0.98)$ & 0.0122 \\
\hline Bladder cancer with confirmation & $85(63)$ & $111(32)$ & $0.93(0.61-1.44)$ & 0.7502 \\
\hline Prostate cancer & 787 (346) & $923(138)$ & $0.82(0.68-0.997)$ & 0.046 \\
\hline Female breast cancer & $609(181)$ & $826(113)$ & $0.85(0.67-1.08)$ & 0.1748 \\
\hline Malignant melanoma & $372(276)$ & $431(124)$ & $0.83(0.67-1.02)$ & 0.0783 \\
\hline Colorectal cancer & $194(144)$ & $326(94)$ & $0.67(0.52-0.86)$ & 0.0021 \\
\hline Non-Hodgkin's lymphoma & 187 (139) & $197(57)$ & $1.15(0.84-1.58)$ & 0.3780 \\
\hline Lung cancer & $169(126)$ & 308 (89) & $0.59(0.45-0.77)$ & 0.0001 \\
\hline Kidney cancer & $103(77)$ & $138(40)$ & $0.87(0.59-1.27)$ & 0.4575 \\
\hline Pancreatic cancer & $67(50)$ & $214(62)$ & $0.30(0.21-0.44)$ & $<0.0001$ \\
\hline Endometrial cancer & $60(45)$ & $100(29)$ & $0.80(0.49-1.30)$ & 0.3612 \\
\hline
\end{tabular}

CI confidence interval, $M I$ myocardial infarction

${ }^{\text {a }}$ HR from Cox regression adjusted with inverse probability of treatment weights

could be declared statistically significant, because the type 1 error $(\alpha)$ had already been 'spent' during the primary endpoint analysis.

\section{Discussion}

This retrospective cohort study used health claims from the i3 InVision Data Mart ${ }^{\mathrm{TM}}$ to conclude that, in a comparison between third-line anti-diabetic agents pioglitazone and insulin, the risk of MI or stroke was $56 \%$ lower in the pioglitazone group, while the risk of nine selected cancers was $22 \%$ lower. Models for bone fracture requiring hospitalization and bladder cancer also yielded hazard ratios in favour of pioglitazone; however, the differences between the two treatment groups were not significant. The fitted Cox regressions used IPT weights derived from propensity scores adjusted for a multitude of confounding variablesincluding age, sex, baseline comorbidities and medications. Various sensitivity analyses confirmed the results.

One particular sensitivity analysis merits special attention. Rather than excluding patients who switched index treatments, or who ever took rosiglitazone, follow-up time was censored either at the point when the patient switched or at the point when the patient started on rosiglitazone. Patients who took rosiglitazone before taking the index medication were again excluded. This scheme added about 7,500 patients to the overall population, of whom roughly 5,500 were in the pioglitazone cohort. This alternative analysis followed an intent-to-treat design and allocated events to the original treatment-for example, patients who were on pioglitazone for a few months before switching to insulin, then a few years later developed cancer, would be counted as pioglitazone events. The effects of the revised design were to slightly increase the pioglitazone incidence rates, slightly lower the insulin incidence rates and shift the hazard ratios somewhat towards the null. The $\mathrm{CV}$ and cancer composite HRs were about 0.10 higher; the bone fracture HR went up by 0.05 and the bladder cancer HR by 0.01. But the results of the hypothesis tests were not altered, and the overall observations and general conclusions remained unchanged.

A recent study that focused on bladder cancer studied two US-based cohorts from the Kaiser Permanente Northern California (KPNC) database, comprising 30,173 patients treated with pioglitazone and 162,926 patients who had never received pioglitazone [14, 15]. Overall, the results showed a lack of association between pioglitazone and the risk of bladder cancer; however, there was a weak association between the risk of bladder cancer and increasing duration of pioglitazone use, as is currently documented on 
Fig. 3 a Kaplan-Meier eventfree probability curves for the composite endpoint of nine cancers; $\mathbf{b}$ raw incidence rates of the composite endpoint of nine cancers. The vertical bars at each year represent pointwise $95 \%$ confidence intervals (CIs) derived from the Poisson distribution, cut off at 3,000 in order to preserve a uniform, reasonable rate scale for all endpoints. $P Y$ person-years
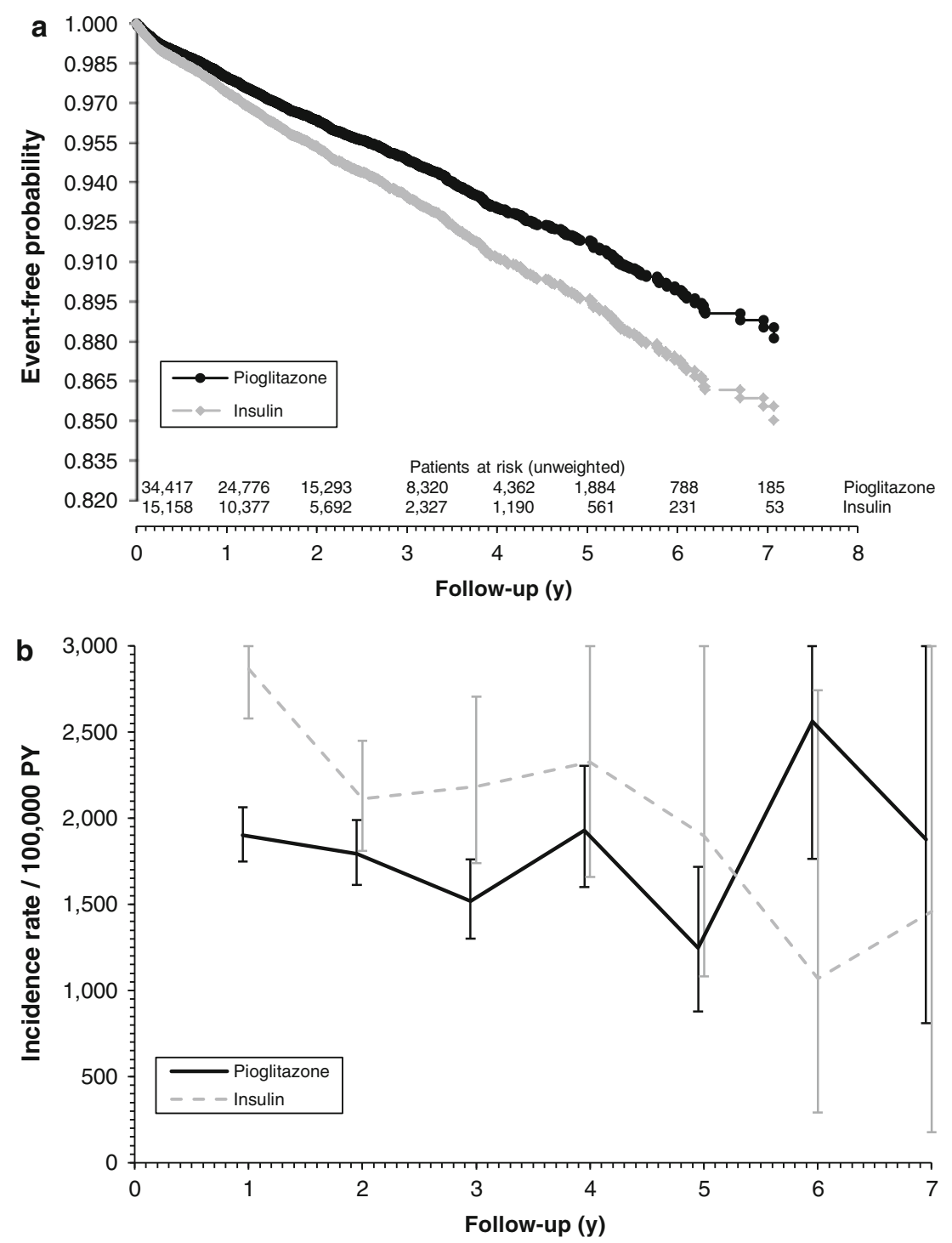

the Actos prescribing information [13]. It should be pointed out that the KPNC study reached its conclusion based on fitted exposure to pioglitazone and that patients on pioglitazone averaged 3.7 years of follow-up, whereas the current study considered raw pioglitazone follow-up time averaging 2.2 years. KPNC registry data showed that the unadjusted incidence rates of bladder cancer in patients who had ever used, or never used, pioglitazone were 81.5 and 68.8 per 100,000 person-years, respectively, closely approximated by the current study's rate of bladder cancer with confirmation (Table 3) and comparable to those published on the Surveillance Epidemiology and End Results (SEER) [30] website for 2000-2008.

Studies performed in European databases have recently appeared. A cohort study of the French national health insurance database found a significant association between exposure to pioglitazone and bladder cancer among 40- to 79-year-olds, albeit with an incidence rate of 49 per 100,000 person-years [16]. A nested case-control study conducted in the UK's General Practice Research Database (GPRD) concluded that while the odds ratio was relatively high, the risk of bladder cancer associated with pioglitazone was low in absolute terms [17]. Propensity score matching was used in a cohort study of diabetic patients in the same GPRD and showed that pioglitazone did not significantly increase the risk of bladder cancer compared with other anti-diabetic medications [18]. The results from the current study also complement recent findings from a cohort study looking at the risk of incident cancer in patients treated with pioglitazone [12]. Insulin is theorized to stimulate cancer cell proliferation and metastasis [31], although the recent ORIGIN (Outcome Reduction with an Initial Glargine Intervention) trial found a neutral relationship between insulin and both cancer and CV outcomes [32].

The event data for MI and stroke in the current study fall in line with those previously published. A retrospective 


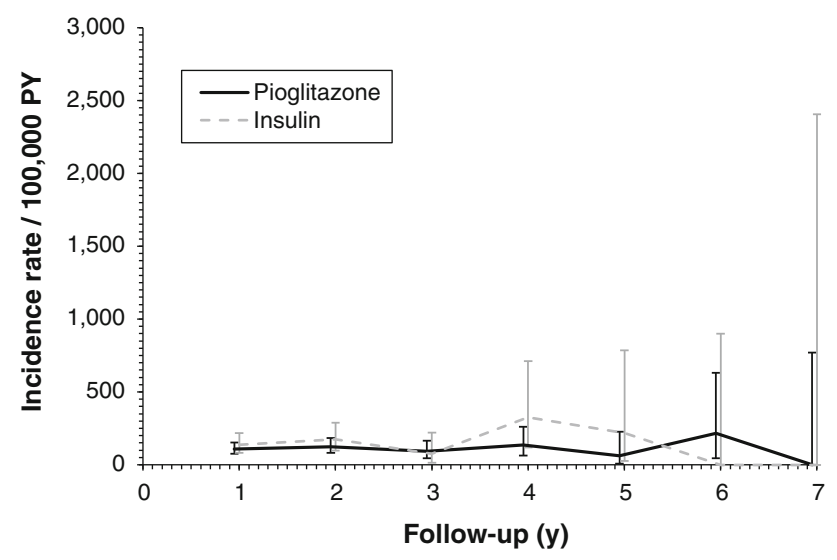

Fig. 4 Raw incidence rates of bladder cancer for pioglitazone and insulin. All incidence rate versus time graphs use the same scale. The vertical bars at each year represent pointwise $95 \%$ confidence intervals (CIs) derived from the Poisson distribution, cut off at 3,000 in order to preserve a uniform, reasonable rate scale for all endpoints. $P Y$ person-years

cohort study, which investigated the risk of AMI during 2003-2006, found that the crude incidence rates of MI were 933 for pioglitazone and 1,113 per 100,000 personyears for rosiglitazone patients [6]. The current study's rate of MI, covering 2003-2010, was 454 per 100,000 personyears (Table 3), in agreement with a recognized-but not well understood - trend of a decline in CV events in recent years [33, 34]. A systematic review of Medline and EMBASE records for randomized, controlled studies of various drugs, with $>1,000$ diabetic subjects, found that the weighted mean incidence rates of MI and stroke were 520 and 540 per 100,000 person-years, respectively [35].

An increased risk of bone fractures is also associated with diabetes [36-41]; a higher incidence of fractures has been reported in patients who were treated with insulin in comparison with T2DM patients who were not [36, 37]. Thiazolidinediones have also been associated with an increased risk of bone fracture [42-44]. A study that used the self-controlled case-series model in the UK compared rates of fracture during TZD exposure with unexposed periods, and found a within-person rate ratio of 1.43 (95\% CI 1.25-1.62) for fracture at any site. This association was similar in men and women, and in patients treated with either rosiglitazone or pioglitazone [42].

The current study required that patients have a baseline period of at least 6 months, enabling a reliable definition of the index medication. However, the relatively short prebaseline period available in the database made it very difficult to reliably estimate the duration of diabetes based on claims. The exclusion of this variable should have been indirectly offset by the extensive list of comorbidity conditions and baseline medications used in the regression model.

This study had other limitations. Lack of randomization entails a risk of confounding due to unbalanced selection of patients, although the Cox model with IPT weights derived from a list of more than 40 confounding variables eliminated some potential bias. The relatively short follow-up average of about 2 years meant that the effects of long-term treatment could not be reliably assessed by this study; such assessments must be left to future, longer-term studies. In addition, patients over 65 years of age may have been missed, as most would have transferred to the US Medicare program and would then have been lost from the claims database, suggesting a potential bias in the current study towards a younger cohort. Also, patients who took both pioglitazone and insulin - who might generally have been in poorer health than the included patients or may have had contraindications to one of the two treatments-were excluded, which led to a slight under-estimation of the incidence rate in the pioglitazone cohort, as demonstrated by a sensitivity analysis.

\section{Conclusion}

This study found that the risks of two composite endpointsone for cardiovascular events, the other for nine cancer sites-were significantly lower among patients exposed to pioglitazone than among those exposed to insulin. While the risks of bone fracture and bladder cancer were lower in the pioglitazone group, the differences were not statistically significant. Among the major endpoints, bladder cancer had by far the lowest risk-one-sixteenth that of the composite of nine cancers - and showed no evidence of its incidence rate increasing with duration of exposure to pioglitazone.

Acknowledgments This study was funded and conducted by Takeda Pharmaceuticals International, Inc. and Takeda Global Research and Development Center, Inc. The sponsors were involved in the design of the study, data collection, analysis and interpretation, the development of this manuscript, and the decision to submit the manuscript for publication. All authors were employed by Takeda while performing and documenting this study. The authors acknowledge Rx Communications (Mold, UK) for providing editorial assistance with the development of the manuscript (funded by Takeda Pharmaceuticals International, Inc). The authors also acknowledge Venkatesh Harikrishnan, an on-site contractor from Ranstad Professionals, for SAS programming support during the study. The authors thank the anonymous reviewers for their valuable suggestions, which improved the manuscript substantially.

Open Access This article is distributed under the terms of the Creative Commons Attribution Noncommercial License which permits any noncommercial use, distribution, and reproduction in any medium, provided the original author(s) and the source are credited.

\section{References}

1. Huxley R, Barzi F, Woodward M. Excess risk of fatal coronary heart disease associated with diabetes in men and women: meta-analysis of 37 prospective cohort studies. BMJ. 2006;332(7533):73-8. 
2. Seshasai SR, Kaptoge S, Thomson A, et al. Emerging Risk Factors Collaboration. Diabetes mellitus, fasting blood glucose, and risk of cause-specific death. New Engl J Med. 2011; 364(9):829-41.

3. Smith U. Pioglitazone: mechanism of action. Int $\mathrm{J}$ Clin Pract Suppl. 2001;121:13-8

4. Goldberg RB, Kendall DM, Deeg MA, et al. GLAI Study Investigators. A comparison of lipid and glycemic effects of pioglitazone and rosiglitazone in patients with type 2 diabetes and dyslipidemia. Diabetes Care. 2005;28(7):1547-54.

5. Deeg MA, Buse JB, Goldberg RB, et al. Pioglitazone and rosiglitazone have different effects on serum lipoprotein particle concentrations and sizes in patients with type 2 diabetes and dyslipidemia. Diabetes Care. 2007;30(10):2458-64.

6. Gerrits CM, Bhattacharya M, Manthena S, et al. A comparison of pioglitazone and rosiglitazone for hospitalization for acute myocardial infarction in type 2 diabetes. Pharmacoepidemiol Drug Saf. 2007;16(10):1065-71.

7. Dormandy JA, Charbonnel B, Eckland DJ, et al. PROactive Investigators. Secondary prevention of macrovascular events in patients with type 2 diabetes in the PROactive Study (PROspective pioglitAzone Clinical Trial In macroVascular Events): a randomised controlled trial. Lancet. 2005;366(9493):1279-89.

8. Currie CJ, Johnson JA. The safety profile of exogenous insulin in people with type 2 diabetes: justification for concern. Diabetes Obes Metab. 2012;14:1-4.

9. Rubenstrunk A, Hanf R, Hum DW, et al. Safety issues and prospects for future generations of PPAR modulators. Biochim Biophys Acta. 2007;1771(8):1065-81.

10. Dai Y, Qiao L, Chan KW, et al. Peroxisome proliferator-activated receptor-gamma contributes to the inhibitory effects of embelin on colon carcinogenesis. Cancer Res. 2009;69(11):4776-83.

11. Ferrara A, Lewis JD, Quesenberry CP, et al. Cohort study of pioglitazone and cancer incidence in patients with diabetes. Diabetes Care. 2011;34(4):923-9.

12. Bowker SL, Majumdar SR, Veugelers P, et al. Increased cancerrelated mortality for patients with type 2 diabetes who use sulfonylureas or insulin. Diabetes Care. 2006;29(2):254-8.

13. Takeda Pharmaceuticals America, Inc. Actos (pioglitazone hydrochloride) tablets: US prescribing information. Deerfield; 2012.

14. Lewis JD, Ferrara A, Peng T, et al. Risk of bladder cancer among diabetic patients treated with pioglitazone: interim report of a longitudinal cohort study. Diabetes Care. 2011;34(4):916-22.

15. Takeda R\&D. Cohort study of pioglitazone and bladder cancer in patients with diabetes: fourth interim analysis (8-year) report, 2012. http://general.takedapharm.com/Trial-Disclosure/01-03TL-OPI-524-8-year-Interim-Report.pdf. Accessed 17 July 2013.

16. Neumann A, Weill A, Ricordeau P, et al. Pioglitazone and risk of bladder cancer among diabetic patients in France: a populationbased cohort study. Diabetologia. 2012;55(7):1953-62.

17. Azoulay L, Yin H, Filion KB, et al. The use of pioglitazone and the risk of bladder cancer in people with type 2 diabetes: nested case-control study. BMJ. 2012;344:e3645.

18. Wei L, MacDonald TM, Mackenzie IS. Pioglitazone and bladder cancer: a propensity score matched cohort study. Br J Pharmacol. 2013;75(1)254-9.

19. Observational Medical Outcomes Partnership. Observational Medical Outcomes Partnership 2011 symposium presentations. http:// omop.fnih.org/OMOP2011Symposium. Accessed 30 Jul 2012.

20. Garber AJ, Abrahamson AJ, Barzilay JI, et al. AACE comprehensive diabetes management algorithm. Endocr Pract. 2013;19(2):327-36.

21. Rosenbaum PR, Rubin DB. The central role of the propensity score in observational studies for causal effects. Biometrika. 1983;70(1):41-55.
22. Joffe MM, Rosenbaum PR. Invited commentary: propensity scores. Am J Epidemiol. 1999;150(4):327-33.

23. Sato T, Matsuyama Y. Marginal structural models as a tool for standardization. Epidemiology. 2003;14(16):680-6.

24. Robins JM, Hernán MA, Brumback B. Marginal structural models and causal inference in epidemiology. Epidemiology. 2000;11(5):550-60.

25. Hernán MA, Brumback B, Robins JM. Marginal structural models to estimate the causal effect of zidovudine on the survival of HIV-positive men. Epidemiology. 2000;11(5):561-70.

26. Choma NN, Griffin MR, Huang RL, et al. An algorithm to identify incident myocardial infarction using Medicaid data. Pharmacoepidemiol Drug Saf. 2009;18(11):1064-71.

27. Roumie CL, Mitchel E, Gideon PS, et al. Validation of ICD-9 codes with a high positive predictive value for incident strokes resulting in hospitalization using Medicaid health data. Pharmacoepidemiol Drug Saf. 2008;17(1):20-6.

28. Lin D, Wei LJ, Ying Z. Checking the Cox model with cumulative sums of martingale-based residuals. Biometrika. 1993;80(3):557-72.

29. Holm S. A simple sequentially rejective multiple test procedure. Scand J Stat. 1979;6:65-70.

30. Surveillance Epidemiology and End Results, National Cancer Institute. Fast stats, 2010. http://seer.cancer.gov/faststats/. Accessed 10 Apr 2012.

31. Giovannucci E, Harlan DM, Archer MC, et al. Diabetes and cancer: a consensus report. CA Cancer J Clin. 2010;60:207-21.

32. The ORIGIN Trial Investigators. Basal insulin and cardiovascular and other outcomes in dysglycemia. New Engl J Med. 2012; 367(4):319-28.

33. Lightwood JM, Glantz SA. Declines in acute myocardial infarction after smoke-free laws and individual risk attributable to secondhand smoke. Circulation. 2009;120(14):1373-9.

34. Yeh RW, Sidney S, Chandra M, et al. Population trends in the incidence and outcomes of acute myocardial infarction. New Engl J Med. 2010;362(23):2155-65.

35. Preiss D, Sattar N, McMurray JJ. A systematic review of event rates in clinical trials in diabetes mellitus: the importance of quantifying baseline cardiovascular disease history and proteinuria and implications for clinical trial design. Am Heart J. 2011;161(1):210.e1-219.e1.

36. Forsén L, Meyer HE, Midthjell K, et al. Diabetes mellitus and the incidence of hip fracture: results from the Nord Trøndelag Health Survey. Diabetologia. 1999;42(8):920-5.

37. Nicodemus KK, Folsom AR, Iowa Women's Health Study. Type 1 and type 2 diabetes and incident hip fractures in postmenopausal women. Diabetes Care. 2001;24(7):1192-7.

38. Schwartz AV, Sellmeyer DE, Ensrud KE, et al. Older women with diabetes have an increased risk of fracture: a prospective study. J Clin Endocrinol Metab. 2001;86(1):32-8.

39. Carnevale V, Romagnoli E, D'Erasmo E. Skeletal involvement in patients with diabetes mellitus. Diabetes Metab Res Rev. 2004;20(3):196-204.

40. de Liefde II, van der Klift M, de Laet CE, et al. Bone mineral density and fracture risk in type-2 diabetes mellitus: the Rotterdam Study. Osteoporos Int. 2005;16(12):1713-20.

41. Janghorbani M, Feskanich D, Willett WC, et al. Prospective study of diabetes and risk of hip fracture: the Nurses' Health Study. Diabetes Care. 2006;29(7):1573-8.

42. Murphy CE, Rodgers PT. Effects of thiazolidinediones on bone loss and fracture. Ann Pharmacother. 2007;41(12):2014-8.

43. Douglas IJ, Evans SJ, Pocock S, et al. The risk of fractures associated with thiazolidinediones: a self-controlled case-series study. PLoS Med. 2009;6(9):e1000154.

44. Aubert RE, Herrera V, Chen W, et al. Rosiglitazone and pioglitazone increase fracture risk in women and men with type 2 diabetes. Diabetes Obes Metab. 2010;12(8):716-21. 